\title{
Bioconversion of Methanol into Value- added Chemicals in Native and Synthetic Methylotrophs
}

\author{
Min Zhang ${ }^{1}$, Xiao-jie Yuan¹, Cong Zhang ${ }^{1}$, Li-ping Zhu', Xu-hua Mo¹, \\ Wen-jing Chen ${ }^{1}$ and Song Yang ${ }^{1,2 *}$
}

\begin{abstract}
${ }^{1}$ School of Life Science, Qingdao Agricultural University, Shandong Province Key Laboratory of Applied Mycology, and Qingdao International Center on Microbes Utilizing Biogas, Qingdao, China.

${ }^{2}$ Key Laboratory of Systems Bioengineering, Ministry of Education, Tianjin University, Tianjin, China.
\end{abstract}

*Correspondence: yangsong1209@163.com

https://doi.org/10.21775/cimb.033.225

\begin{abstract}
Methanol, commercially generated from methane, is a renewable chemical feedstock that is highly soluble, relatively inexpensive, and easy to handle. The concept of native methylotrophic bacteria serving as whole cell catalysts for production of chemicals and materials using methanol as a feedstock is highly attractive. In recent years, the available omics data for methylotrophic bacteria, especially for Methylobacterium extorquens, the best-characterized model methylotroph, have provided a solid platform for rational engineering of methylotrophic bacteria for industrial production. In addition, there is a strong interest in converting the more traditional heterotrophic production platforms towards the use of single carbon substrates, including methanol, through metabolic engineering. In this chapter, we review the recent progress towards achieving the desired growth and production yields from methanol, by genetically engineered native methylotrophic strains and by the engineered synthetic methylotrophs.
\end{abstract}

\section{Introduction}

One goal of metabolic engineering is to develop renewable and sustainable alternatives in production of value-added chemicals. In particular, methanol is an important one-carbon (C1) feedstock that can be generated from either synthesis gas (a mixture of $\mathrm{CO}$ and $\mathrm{H}_{2}$ ) or from biogas, assuming that large quantities of this feedstock could be produced at relatively low market price (Clomburg et al., 2017; Yang et al., 2018). Methylotrophic bacteria are a diverse group of microbes that can use reduced $\mathrm{C} 1$ compounds such as methanol and methane as sole sources of both energy and carbon (Chistoserdova et al., 2009; Chistoserdova, 2018). Some methylotrophic bacteria, such as Methylobacterium extorquens, Methylococcus capsulatus, and Methylomicrobium buryatense, show robust growth under laboratory conditions (Bird et al., 1971; Guo and Lidstrom, 2006; de la Torre et al., 2015). The availability of whole genome sequences, combined with biochemical studies (Peyraud et al., 2011; Kalyuzhnaya et al., 2013), have provided important insights into their metabolism that are crucial for enabling engineering these types of microorganism for bulk chemical production. Meantime, as an alternative approach, more traditional model microorganisms such has Escherichia coli, Corynebacterium glutamicum and Saccharomyces cerevisiae, have been genetically modified towards acquiring the ability to utilize methanol for growth, 
with a potential for converting methanol into valueadded compounds (Whitaker et al., 2015; Bennett et al., 2018b). In this chapter, we review the current status of the fields of engineering native as well as synthetic methylotrophs and provide examples of successfully engineered strains with a potential in producing commercial value-added chemicals.

\section{Metabolic engineering of native methylotrophs for value-added chemicals production}

M. extorquens AM1, a pink-pigmented facultative methylotrophic $\alpha$-proteobacterium capable of growth on both $\mathrm{C} 1$ and multi-carbon compounds, has served for decades as the model organism for studying $\mathrm{C} 1$ metabolism (Chistoserdova et al., 2003), including its biotechnological applications (Ochsner et al., 2015). Since the 1960s, it has been reported that $M$. extorquens was successfully employed as a producer of certain products such as amino acids (Sirirote et al., 1988). Based on the current understanding, $M$. extorquens employs three interlocked metabolic cycles for carbon assimilation: the serine cycle, the ethylmalonyl-CoA (EMC) pathway, and the poly-3-hydroxybutyrate (PHB) cycle (Fig. 13.1) (Anthony, 2011; Cui et al., 2016). The main function of the EMC pathway is to regenerate glyoxylate from acetyl-CoA for reincorporating it into the serine cycle during $\mathrm{C} 1$ assimilation (Erb et al., 2007; Peyraud et al., 2009). It has been shown that significant metabolic flux goes through the serine cycle and the EMC pathway when cells are grown on methanol, generating a stable supply of acetyl-CoA, which could serve as a precursor for the production of value-added chemicals in engineered $M$. extorquens AM1 strains (Fig. 13.1) (Peyraud et al., 2011; Fu et al., 2016).

\section{Production of mevalonate and terpenoids}

Mevalonate and its derivatives terpenoids are promising bulk chemicals. Terpenoids are used as flavours, fragrances, pharmaceuticals, and biofuels, representing the largest and the most structurally diverse family of chemicals in nature (Zhang et al., 2017). To synthesize considerable amounts of terpenoids, a commonly used strategy in bacteria is to introduce an exogenous mevalonate (MEV) pathway instead of utilizing the native methylerythritol phosphate (MEP) pathway for circumventing the regulatory control of the MEP pathway (Zhao et al., 2013). Thus, pathway engineering for mevalonate production is a feasible first step as a platform for diverse terpenoid synthesis. Although E. coli is the primary industrial microbe for the production of mevalonate (Nagai et al., 2018), other engineered hosts have the potential to generate it from alternative carbon feedstocks via synthetic metabolic pathways. Zhu and colleagues

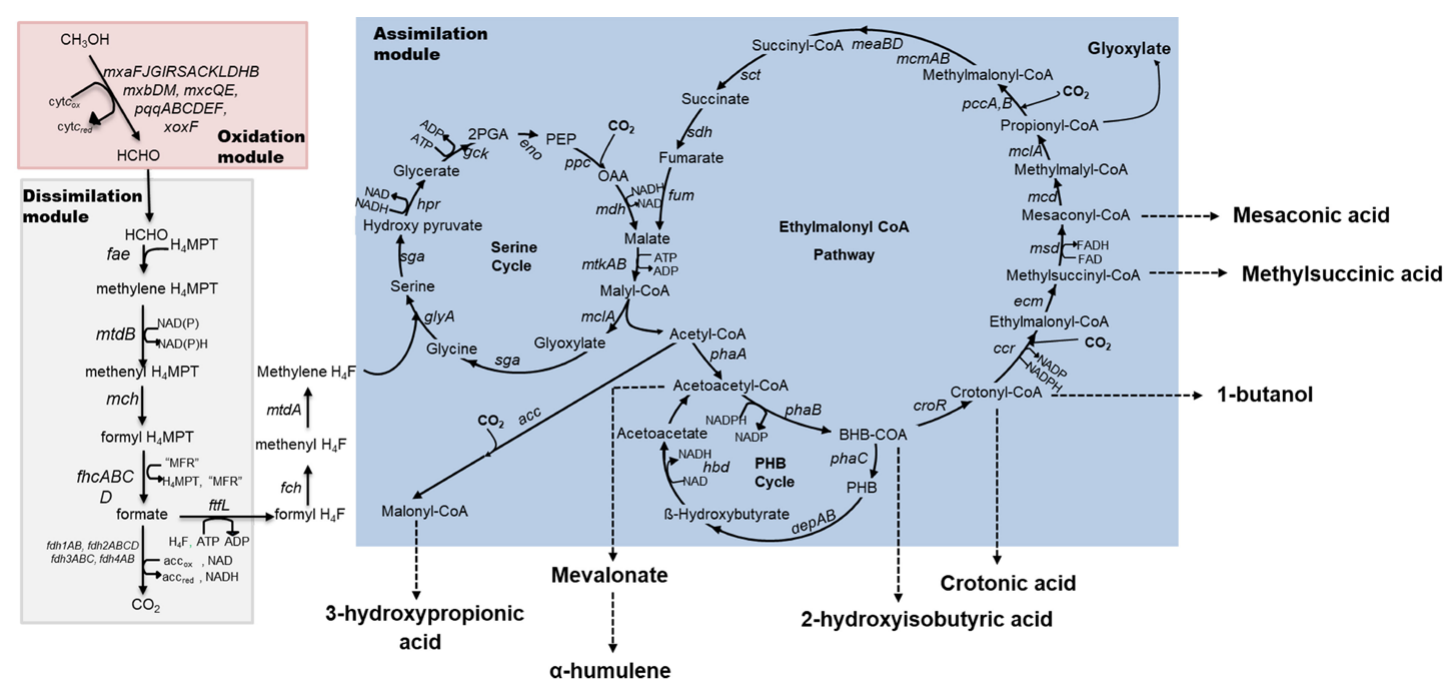

Figure 13.1 Schematic of methanol metabolism in $M$. extorquens and target precursors for synthesis of value added compounds. Pink, grey and blue backgrounds represent the primary oxidation module, the dissimilation module, and the assimilation modules, respectively. 
engineered a mevalonate pathway by using acetyl$\mathrm{CoA}$ as the initial precursor in M. extorquens AM1, to produce mevalonate from methanol (Zhu et al., 2016). A natural operon (MVH) harbouring the mvaS and mvaE genes from Enterococcus faecalis, as well as an artificial operon (MVH) harbouring the hmgcs 1 gene from Blattella germanica and the tchmgr gene from Trypanosoma cruzi were constructed in a plasmid pCM110. Expression of these two operons resulted in the titres of mevalonate of $56 \mathrm{mg} / 1$ and $66 \mathrm{mg} / \mathrm{l}$, respectively, in flask cultivations. Further introduction of the phaA gene from Ralstonia eutropha into the MVH operon, with the goal of improving the conversion from acetyl-CoA into acetoacetyl-CoA increased the mevalonate titre to $180 \mathrm{mg} / \mathrm{l}, 3.2$-fold higher than that of the natural MVE operon. Further modification of the expression level of the phaA gene by regulating the strength of the ribosomal binding site (RBS) resulted in an additional increase in mevalonate production to $215 \mathrm{mg} / \mathrm{l}$ (Zhu et al., 2016). Moreover, it has been demonstrated that, under these conditions, the supply of acetyl-CoA was limited in engineered $M$. extorquens AM1. To overcome this limitation, a strategy termed mevalonate sensor-assisted transcriptional regulator engineering (SATRE) was employed, to control metabolic flux redistribution toward increasing acetyl-CoA flux from methanol for mevalonate production (Fig. 13.2) (Liang et al., 2017a). A 60\% increase in mevalonate concentration was further achieved following sensor-based high-throughput screening of a QscR transcriptional regulator library. Finally, a fed-batch fermentation in a 51 bioreactor yielded a mevalonate concentration of $2.67 \mathrm{~g} / \mathrm{l}$, which was equivalent to an overall yield of $0.055 \mathrm{~mol}$ acetyl$\mathrm{CoA} / \mathrm{mol}$ methanol. In addition, Cui and colleagues utilized atmospheric and room temperature plasma (ARTP) mutagenesis, in combination with adaptive laboratory evolution (ALE), generating a mutant with high methanol tolerance whose cell density was 7.1-fold higher than that of the parent strain in $1.25 \mathrm{M}(5 \%, \mathrm{v} / \mathrm{v})$ methanol (Cui et al., 2018). Accordingly, the mevalonate productivity of the mutant strain carrying a mevalonate synthesis pathway was $65 \%$ higher than that of the wild-type strain in methanol fed-batch fermentation.

In a separate study, Sonntag and colleagues introduced a mevalonate pathway from Myxococcus xanthus, in combination with RBS optimization of $\alpha$-humulene and farnesyl pyrophosphate synthases, resulting in the increase of titres of $\alpha$-humulene from $18 \mathrm{mg} / 1$ to $54 \mathrm{mg} / 1$ in M. extorquens AM1 (Sonntag et al., 2015a). Furthermore, the recombinant M. extorquens AM1 was grown in methanol-limited fed-batch conditions, with the addition of dodecane to avoid the accumulation of $\alpha$-humulene in the medium. An average $\mathrm{OD}_{600}$ value of $80-90$ were achieved, and final product concentrations were up to $1.65 \mathrm{~g} / \mathrm{l}$. This is the highest titre of $d e$

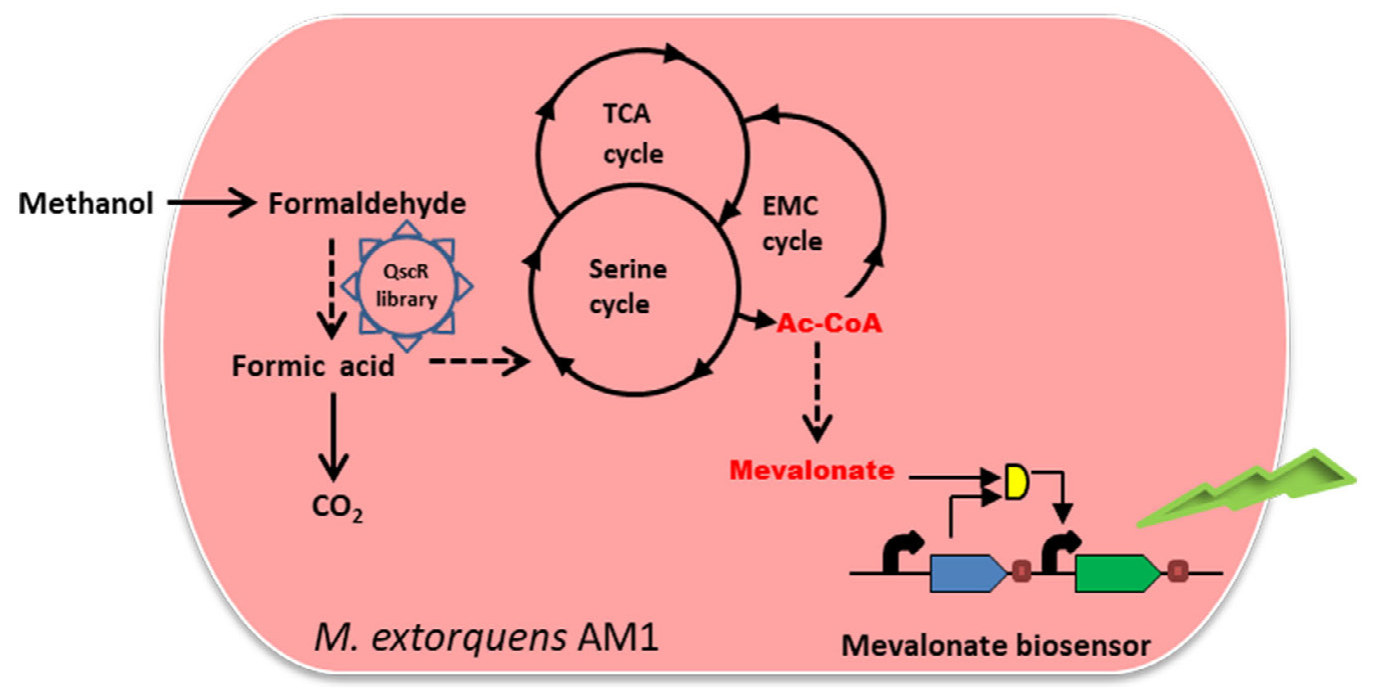

Figure 13.2 Biosensor-assisted transcriptional regulator engineering approach to increase mevalonate production in M. extorquens AM1. QscR is the regulator of the serine cycle for methanol assimilation (Liang et al., 2017). 
novo synthesized $\alpha$-humulene reported to date, which corresponds to $12 \%$ of the maximum theoretical yield. The product titres of mevalonate and $\alpha$-humulene described in these studies are already in a competitive range compared to the industrial platform strains (Harada et al., 2009), demonstrating the potential of $M$. extorquens as a future platform for the production of high-value terpenoids.

\section{Production of carboxylic acids}

3-Hydroxypropionic acid (3-HP) is an important platform chemical, serving as a precursor for a wide range of industrial applications, such as the production of acrylic acid and 1,3-propanediol. In particular, acrylic acid has an annual market exceeding $\$ 10$ billion. 3-HP can be further polymerized into poly-3-hydroxypropionate, which is a biodegradable and biocompatible thermoplastic polymer. Recently, Yang and colleagues constructed a malonyl-CoA pathway in M. extorquens AM1 that reduced malonyl-CoA to synthesize 3-HP through a bifunctional reductase encoded by the $\mathrm{mor}$ gene from Chloroflexus aurantiacus (Yang et al., 2017). The engineered strain demonstrated 3-HP production from methanol with initial titre of $6.8 \mathrm{mg} / \mathrm{l}$ in shake flask cultivation, which was then improved to $91.0 \mathrm{mg} / \mathrm{l}$ by increasing the strength of the promoter and the $m c r$ gene copy number, and by decreasing the concentration of sodium ions in the culture medium. Notably, when the cells reached the stationary growth phase, 3-HP content was rapidly reduced to lower than $10.0 \mathrm{mg} / \mathrm{l}$ within $10 \mathrm{~h}$. Furthermore, based on the combined results from in vitro enzyme assays, ${ }^{13} \mathrm{C}$-labeling analysis, metabolomics and gene knockouts, major routes were identified responsible for the degradation of the 3-HP during this phase of growth. This work provides a solid base for future engineering efforts towards bioconversion of methanol into the economically important product 3-HP.

Recently, Rohde and colleagues introduced (R)-3-hydroxybutyryl-CoA-specific, coenzyme B12-dependent mutases from Bacillus massiliosenegalensis JC6 into $M$. extorquens AM1, to convert the intermediate of 3-hydroxybutyrl-CoA towards production of another carboxylic acid, 2-hydroxyisobutyric acid, a building block with a high potential for poly-methylmethacrylate production (Rohde et al., 2017). 2-hydroxyisobutyric acid titres of $2.1 \mathrm{~g} / 1$ were further achieved in fed-batch bioreactor without affecting the biomass yield, with the yield of $0.11 \mathrm{~g} / \mathrm{g}$ from methanol. In addition, Sonntag and colleagues overexpressed $y c i A$ encoding thioesterases, from E. coli in M. extorquens AM1, an engineering step designed to generate two dicarboxylic acids from their CoA derivatives involved in the EMC pathway, mesaconic acid (with $70 \mathrm{mg} / 1$ yield), and methylsuccinic acid (with $60 \mathrm{mg} / \mathrm{l}$ yield), respectively (Sonntag et al., 2014). Further increase in accumulation of mesaconyl-CoA (16-fold) and methylsuccinyl-CoA (6-fold) was achieved by adjusting the concentration of cobalt, a growth limiting factor affecting the activities of ethylmalonyl-CoA and methylmalonyl-CoA mutases, in the culture medium, with a combined titre of the two dicarboxylic acids of $0.65 \mathrm{~g} / 1$ (Sonntag et al., $2015 \mathrm{~b}$ ). This titre represented a yield of $0.17 \mathrm{~g} / \mathrm{g}$ methanol. Not only these important achievements shed light on the potential in engineering $M$. extorquens AM1 as an alternative microbial factory for producing common carboxylic acids and uncommon dicarboxylic acids from methanol, but they also illustrate the complex metabolic regulation of methylotrophy in this organism.

\section{Production of 1-butanol}

1-Butanol has been proposed to be a better alternative to ethanol as a replacement for gasoline because of its many advantages. For instance, 1-butanol is less soluble in water than ethanol, reducing the possibility of contamination in groundwater, and this trait also makes it feasible to use liquid-liquid extraction for 1-butanol recovery in industrial fermentation. Additionally, 1-butanol is compatible with the current petroleum infrastructure and can be blended with gasoline at high ratios. Although clostridia are the primary industrial microbes for the fermentative production of 1-butanol, clostridia-based industrial fermentation has some limitations, such as formation of by-products, spore formation, and low cell density (Lee et al., 2008). Recently, $\mathrm{Hu}$ and colleagues engineered a modified CoA-dependent pathway for 1-butanol production from the precursor crotonyl-CoA, by introducing the ter gene from Treponema denticola and the adhE2 from Clostridium acetobutylicum into M. extorquens AM1 (Hu and Lidstrom, 2014). Although tests for 1-butanol production by cells grown on methanol were negative, the engineered strain was able to generate 1 -butanol at $15.2 \mathrm{mg} / 1$ when grown on 
ethylamine while overexpressing the $c r o R$ gene as a means of increasing the supply of crotonyl-CoA (Hu and Lidstrom, 2014). Adaptive laboratory evolution (ALE) was further employed, resulting in isolation of a mutant with 1-butanol tolerance of up to $0.5 \%$, after 200 generations. The adapted strain was able to produce $25.5 \mathrm{mg} / 1$ 1-butanol, representing an $87 \%$ increase in production compared with the original strain (Hu et al., 2016). Whole genome sequencing revealed a SNP mutation in the adapted strain, in $k e f B$, a gene encoding a potassium/proton antiporter. Analysis of a knockout mutant in this gene confirmed that this mutation was indeed responsible for the increased 1-butanol tolerance. Global metabolomics analysis further uncovered that the pools of multiple key metabolites, including fatty acids, amino acids, disaccharides and carotenoids were either increased or decreased in the adapted strain in response to 1-butanol stress. Overall, this study uncovered an array of cellular responses by $M$. extorquens AM1 to solvent stresses, thus providing a genetic blueprint for further rational design of derivatives of this strain for increased 1-butanol production.

\section{Novel insights into methylotrophy in Methylobacterium}

Although natural methylotrophs represent promising candidates as new microbial cell factories, further efforts are required to better understand the regulation of methylotrophs in these species and to further develop genetic tools essential for improving the product yields. Recently, Ochsner and colleagues carried out experiments involving hyper-saturating transposon mutagenesis, followed up by high-throughput sequencing of mutant populations (Ochsner et al., 2017). This study uncovered 95 genes that have not been previously known to play a role in methylotrophy (Bringel and Vuilleumier, 2017; Ochsner et al., 2017). Notably, this study uncovered that ribulose-1, 5-bisphosphate (RuBP) functions as a master regulator metabolite in methylotrophic metabolism, by uncovering that prk, a gene encoding phosphoribulokinase (Prk), responsible for the production of RuBP was essential for growth on methanol. Prk was ultimately demonstrated to be a co-inducer of QscR, a major regulator of the serine cycle.

In another study, Schada von Borzyskowski and colleagues explored the possibility of converting the heterotrophic M. extorquens AM1 into an autotrophic derivative (Schada von Borzyskowski et al., 2018). They constructed a chassis that utilized methanol solely for energy acquisition while forming the biomass entirely from $\mathrm{CO}_{2}$. First, they engineered a $M$. extorquens AM1 derivative with deletion in $f t f l$, a gene encoding formate tetrahydrofolate ligase, causing the inability to grow on methanol. They then heterologously expressed Rubisco from Rhodospirillum rubrum, in order to enable $\mathrm{CO}_{2}$ fixation. As a result, $M$. extorquens $\mathrm{AM} 1 \Delta f t f L$ expressing the newly built Calbin-Benson-Bassham (CBB) cycle could grow in methanol minimal medium with $5 \% \mathrm{CO}_{2}$, although this strain did not sustain long-lasting growth under this condition. This work is the first example of decoupling energy metabolism and carbon assimilation in M. extorquens AM1 and establishing synthetic autotrophy with a heterologously derived CBB cycle. This work also demonstrated the importance of the continuous operation of carbon assimilatory cycles in balancing carbon fluxes between biomass building and draining metabolites from central metabolism into the designed biosynthetic pathways. Taken together, these new contributions offer opportunities for elucidating genotype to phenotype relationships in $\mathrm{C}_{1}$ assimilation and providing novel insights into its regulation, which will be key in engineering native methylotrophs for biotechnological application in the future.

\section{Constructing synthetic methylotrophs for direct methanol conversion}

In recent years, there has been increasing interest in engineering non-methylotrophic bacteria toward the use of $\mathrm{C} 1$ compounds, through metabolic engineering (Bennett et al., 2018b). This strategy not only increases our fundamental understanding of methylotrophy, but also provides an opportunity to use established industrial bacteria as platforms for converting methanol into value-added products. The efforts to engineer methanol utilization in traditional platform organisms, both prokaryotic (E. coli, C. glutamicum; Müller et al., 2015b; Witthoff et al., 2015) and eukaryotic S. cerevisiae (Dai et al., 2017) have been recently reported. 


\section{Overview of assimilatory $\mathrm{C}_{1}$ metabolism}

Generally, there are two types of methanol dehydrogenase. One is PQQ-dependent Mdh in Gram-negative methylotrophs such as $M$. extorquens, another is NAD-dependent Mdh in Gram-positive Bacillus methanolicus (Müller et al., 2015a). At least 14 gene products are necessary for PQQ-dependent Mdh function (Lidstrom, 2006), but NAD-dependent Mdh requires one gene for converting methanol to formaldehyde, and its activity can be greatly enhanced by an activator protein ACT (Anne et al., 2013). Moreover, the PQQ cofactor that is required for PQQ-Mdh is not synthesized by the industrial strains such as E. coli or C. glutamicum. Therefore, NAD-dependent Mdh has become an obvious choice for implementation of methanol to formaldehyde conversion in E. coli and C.glutamicum (Fig. 13.3).

For assimilation of formaldehyde into central metabolism, two major pathways in natural methylotrophs have been reported, the ribulose monophosphate (RuMP) and the serine cycles. The RuMP cycle is present in several methylotrophs such as Methylococcus capsulatus Bath and Methylobacillus flagellatus KT (Strom et al., 1974; Chistoserdova et al., 2000). Formaldehyde is condensed with RuMP by hexulose-6-phosphate synthase (HPS), to generate hexulose-6-phosphate. Hexulose-6-phosphate is further isomerized into fructose 6-phosphate by phosphohexuloisomerase (PHI). Fructose 6-phosphate can be cleaved into $\mathrm{C}_{3}$ intermediates either via the glycolysis pathway or via the Entner-Doudoroff pathway reactions. The RuMP pathway is quite an efficient route for formaldehyde assimilation in terms of ATP consumption and biomass yield. Per one molecule of pyruvate generated, 1 molecule of ATP and 3 molecules of $\mathrm{NAD}(\mathrm{P}) \mathrm{H}$ are produced.

The serine cycle operates in methylotrophic bacteria such as M. extorquens AM1 (Chistoserdova and Lidstrom, 1994). In this cycle, formaldehyde is first oxidized to formate, which is subsequently converted into methylene tetrahydrofolate (THF) via the THF-linked $\mathrm{C}_{1}$ transfer pathway. Methylene-THF is then condensed with glycine to produce serine, which is subsequently transformed to form $\mathrm{C}_{3}$ and $\mathrm{C}_{4}$ intermediates for biomass synthesis. In contrast to the RuMP cycle, the serine cycle leads to the consumption of four molecules of
$\mathrm{NAD}(\mathrm{P}) \mathrm{H}$ and three molecules of ATP to produce one molecule of pyruvate.

\section{Engineering bacteria for methanol conversion}

In silico modelling predicted that expression of a NAD-linked Mdh and the two first enzymes of the RuMP cycle, Hps and Phi, into a non-methylotroph such as E. coli should be sufficient to allow biomass formation from methanol (Müller et al., 2015b). In order to test this hypothesis, Müller and colleagues heterologously expressed respective genes in E. coli (Fig. 13.3) (Müller et al., 2015b). The catalytic activities of several candidate enzymes from different microorganisms such as $B$. methanolicus MGA3, Lysinibacillus fusiformis, M. flagellatus KT, $M$. capsulatus Bath were compared in cell lysates, and subsequently in vivo by measuring formaldehyde formation and assimilation. The three genes originating from B. methanolicus MGA3 were found to be the most effective in, respectively, methanol oxidation and formaldehyde conversion by E. coli. To further demonstrate how the metabolism of E. coli was affected by the introduction of the $\mathrm{C} 1$ modules, dynamic labelling experiments using ${ }^{13} \mathrm{C}$-labelled methanol were performed in an E. coli strain lacking the frmA gene that is responsible for formaldehyde detoxification in native E. coli. The incorporation of ${ }^{13} \mathrm{C}$ into hexose 6-phosphates was measured to be within approximately $40 \%$. Other metabolites of the RuMP cycle, such as ribose 5-phosphate and ribulose 5-phosphate, as well as intermediates of the glycolysis pathway, such as dihydroxyacetone phosphate and phosphoenolpyruvate were detected as well, demonstrating that at least several turnovers have occurred through the synthetic RuMP cycle. However, no methanol-dependent growth of $E$. coli was achieved. Meantime, a similar engineering scheme has also been applied to C. glutamicum, demonstrating utilization of methanol as a co-carbon source in a glucose-based medium in (Lessmeier et al., 2015; Witthoff et al., 2015).

Since Mdh from B. methanolicus has low affinity toward methanol ( $\mathrm{Km}$ of $200 \mathrm{mM})$, Whitaker and colleagues employed and alternative enzyme from B. stearothermophilus, possessing a $\mathrm{Km}$ of $20 \mathrm{mM}$ and $V_{\max }$ of $2.1 \mathrm{U} / \mathrm{mg}$ for methanol (Price et al., 2016; Whitaker et al., 2017). They engineered an E. coli strain expressing B. stearothermophilus Mdh 


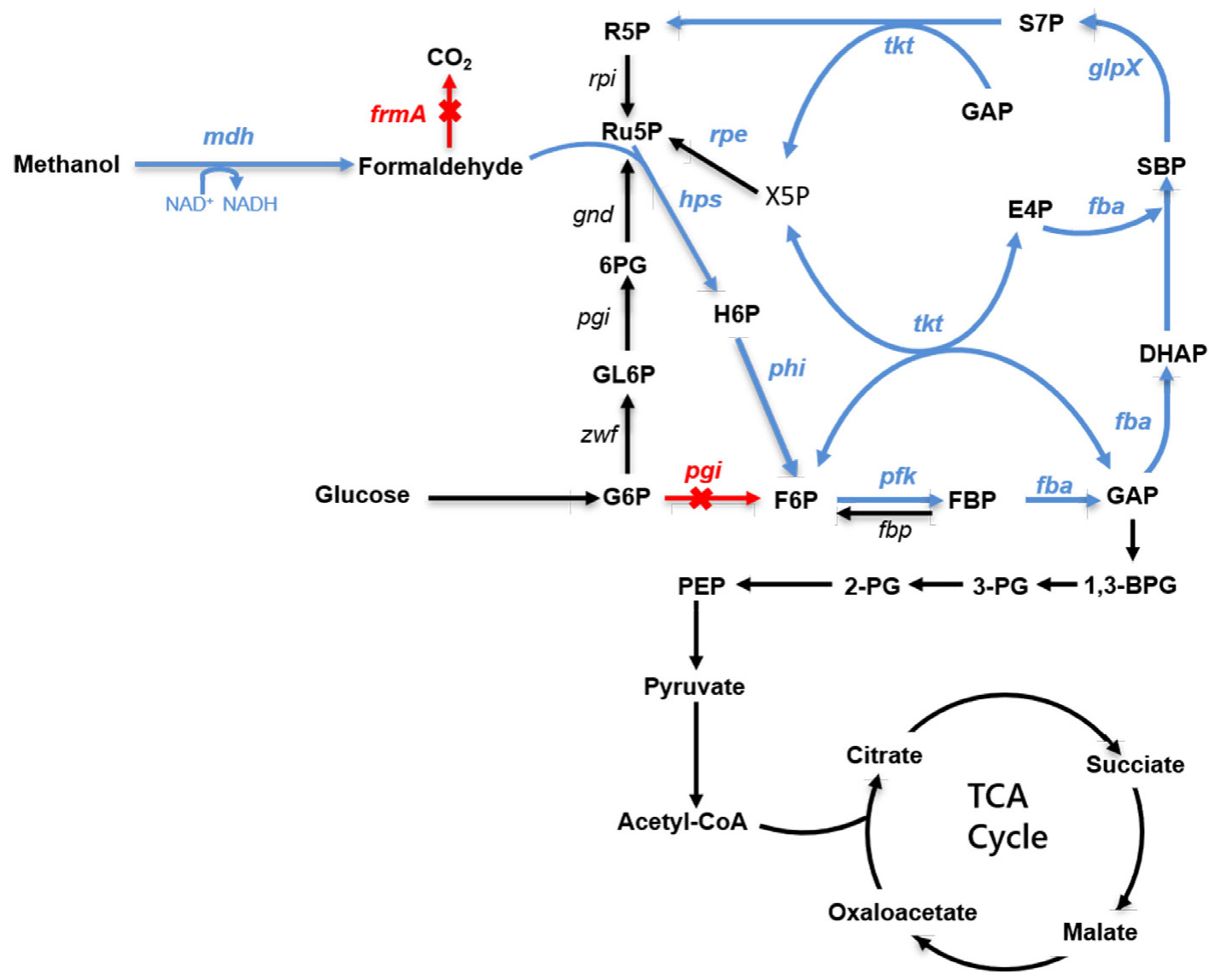

Figure 13.3 Methanol metabolism in synthetic methylotrophs. Blue solid lines represent reactions mediated by the heterologously expression genes. Red solid lines represent gene knockouts. Enzymes (genes): methanol dehydrogenase $(m d h)$, hexulose phosphate synthase (hps), phosphohexuloisomerase (phi), phosphoglucoisomerase $(\mathrm{pg} i)$, formaldehyde dehydrogenase $(\mathrm{frmA})$, phosphofructokinase ( $\mathrm{ffk}$ ), fructosebisphosphate aldolase (fba), transketolase (tkt), ribulose phosphate epimerase (rpe), sedoheptulose bisphosphatase $(g / p X)$, fructose bisphosphatase $(f b p)$, ribose phosphate isomerase (rpi), glucose-6phosphate dehydrogenase (zwf). Metabolites: glucose 6-phosphate (G6P), 6-phosphogluconolactone (GL6P), 6-phosphogluconate (6PG), ribulose 5-phosphate (Ru5P), ribose 5-phosphate (R5P), hexulose 6-phosphate $(\mathrm{H} 6 \mathrm{P})$, fructose 6-phosphate (F6P), fructose-1, 6-bisphosphate (FBP), dihydroxyacetone phosphate (DHAP), glyceraldehyde 3-phosphate (GAP), xylulose 5-phosphate (X5P), erythrose 4-phosphate (E4P), sedoheptulose bisphosphate(SBP), sedoheptulose 7-phosphate (S7P), 1,3-bisphosphoglycerate(1,3-BPG), 3-phosphoglycerate (3-PG), 2-phosphoglycerate (2-PG), phosphoenolpyruvate (PEP)

and B. methanlicus Hps and Phi, and found that small amounts of supplements such as yeast extract enabled initiation of growth of E. coli on methanol. When the engineered $E$. coli strain was grown in M9 minimal medium with $1 \mathrm{~g} / 1$ yeast extract and $60 \mathrm{mM}$ methanol, a cell biomass increase by $30 \%$ was observed, corresponding to biomass yield of $0.289 \mathrm{gCDW} / \mathrm{g}$ methanol. This yield is close to biomass yields reported for native methylotrophs. ${ }^{13} \mathrm{C}$ labelling analysis demonstrated that not only the RuMP cycle intermediates were labelled, but also the metabolites in the TCA cycle and related amino acids, supporting biomass generation from methanol. Moreover, in this study, a $65 \%$ improvement in flavonoid production was observed in the presence of $60 \mathrm{mM}$ methanol. Additional engineering effort included heterologous expression of the non-oxidative pentose phosphate pathway (PPP) consisting of five enzymes (ribulose phosphate epimerase (rpe), fructose-bisphosphate aldolase $(f b a)$, sedoheptulose bisphosphatase $(g l p X)$, phosphofructokinase $(p f k)$ and transketolase $(t k t)$ 
(Bennet et al., 2018a). The introduction of the five genes, all originating from $B$. methanolius, resulted in increased flux through the PPP pathway, further enhancing methanol assimilation by the engineered E. coli (Fig. 13.3) (Bennett et al., 2018a). In addition, Bennett and colleagues deleted the phosphoglucoisomerase gene ( $p g i)$, further increasing carbon flux through the oxidative PPP, for increased supply of Ru5P. This change led to a significant improvement of methanol assimilation and to increased acetone titres during co-culturing on methanol and glucose.

In order to further increase methanol consumption, Meyer and colleagues used genetic constraints to develop an approach to link methanol assimilation to growth supported by a multi-carbon source (Meyer et al., 2018). Via in silico analysis of a metabolic scenario including the presence of the $m d h$-hps-phi operon, they predicted that the deletion of $e d d$, encoding phosphogluconate dehydratase, and rpiAB, encoding ribose-5-phosphate isomerase, would resulted in failure to grow on gluconate, but this variant would growth in the presence of methanol. ALE was then employed to improve co-consumption of methanol and gluconate. After 35 generations, the adapted strain showed 3-fold increase in final $\mathrm{OD}_{600}$, compared with the initially engineered strain. Furthermore, deletion of the NAD-dependent malate dehydrogenase (maldh), which is involved in the TCA cycle, was predicted to increase the ratio of $\mathrm{NAD}+$ / $\mathrm{NADH}$, potentially increasing methanol oxidation and methanol dependency. After additional rounds of ALE of the $\triangle e d d \triangle r p i A B \Delta$ maldh variant of E. coli, growth rate was further improved, from $0.017 / \mathrm{h}$ to $0.081 / \mathrm{h}$ when during co-cultivated on methanol and gluconate. This evolved strain had a methanol consumption rate of $13 \mathrm{mmol} / \mathrm{gCDW} / \mathrm{h}$, which is comparable with the reported rate for M. extorquens AM1. This work provided an important basis for further optimizing synthetic methylotrophic organisms, in the future.

\section{Engineering a formaldehyde- inducible promoter for improved growth on methanol}

In synthetic methylotrophs, methanol is converted to the toxic intermediate formaldehyde by Mdh, catalyzing the initial reaction of methanol conversion. E. coli has a native formaldehyde-inducible promoter, $P$ frm, which negatively regulates the formaldehyde detoxification operon frmRAB (Fig. 13.4) (Danilov and Ismailov, 1989). Recently, Rohlhill and Sandoval constructed a library of mutant variants of Pfrm and used the combination of fluorescence-activated cell sorting and high-throughput sequencing (sort-seq) to screen formaldehyde-responsive promoters (Rohlhill and Sandoval, 2017). Two 19 base pair repressor binding sites, one upstream of the -35 RNA polymerase (RNAP) binding site and one overlapping with the -10 site were identified and subsequently engineered, resulting in 13-fold higher expression compared with the native promoter. They also found that the mutant Pfrm, placed upstream of the methanol assimilation module (i.e. the $m d h$ hps-phi operon), resulted in increased biomass from methanol, likely due to the improved balance in formaldehyde production/consumption. Further, Woolston and colleagues rationally designed the sequence between -10 and -35 region of the Pfrm, and found that the engineered promoter possessed increased sensitivity and wider dynamic range of sensing formaldehyde, at levels as low as $1 \mu \mathrm{M}$. This synthetic biosensor was also employed for optimizing the expression levels of $m d h$ and $h p s /$ phi, to maximize the methanol assimilation flux (Woolston et al., 2018).

\section{Preliminary test for direct methanol utilization in yeast}

Besides prokaryotic methylotrophs, S. cerevisiae also presents a great potential as a microbial chassis for methanol conversion, due to its robust tolerance to high concentrations of methanol. Dai and colleagues integrated a methanol oxidation pathway including alcohol oxidases (AOX), catalases (CAT), dihydroxyacetone synthases (DAS1/2), and dihydroxyacetone kinase (DAK), originating from the methylotrophic yeast Pichia pastoris, into the chromosome of S. cerevisiae (Dai et al., 2017). These methylotrophic enzymes with targeting signals from $P$. pastoris were found to be functionally expressed and located in the peroxisomes in the recombinant $S$. cerevisiae. The engineered $S$. cerevisiae showed $1.04 \mathrm{~g} / \mathrm{l}$ consumption of methanol and $3.13 \%$ increase in cell growth, accompanied with $0.26 \mathrm{~g} / \mathrm{l}$ of pyruvate production in the fermentation broth when using methanol as the sole carbon source. In another study, P. pastoris was engineered to produce lovastatin and its precursor monacolin 
(A)
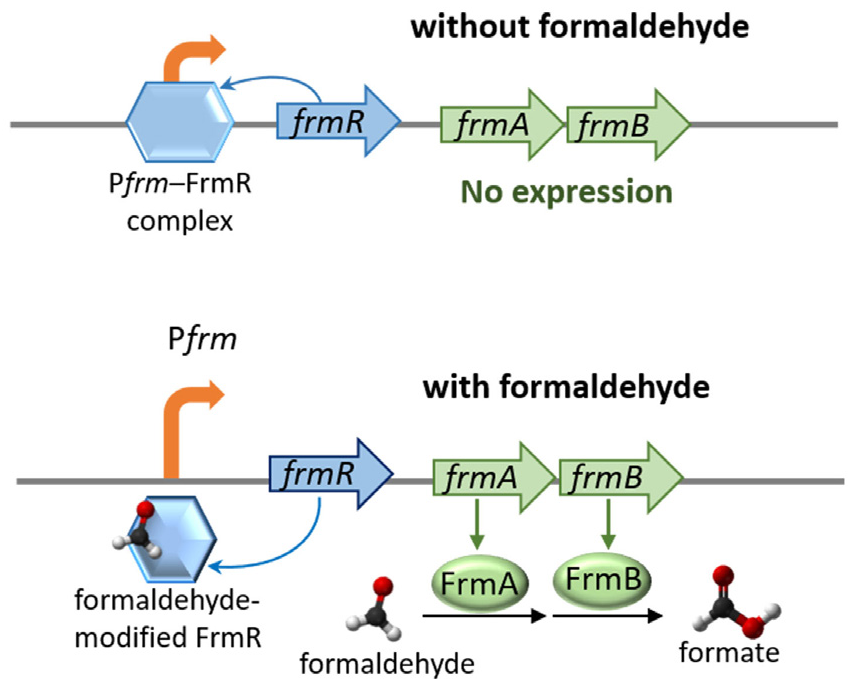

Figure 13.4 Regulation of the fmr operon. (A) FrmR binds to the promoter (Pfrm) upstream of the frmRAB gene cluster, repressing transcription. (B) FrmR is conformationally modified by binding formaldehyde, permitting transcription.

J from methanol (Liu et al., 2018a,b). A co-culture of $P$. pastoris was optimized for bioreactor fermentations with the yields of $593.9 \mathrm{mg} / \mathrm{l}$ monacolin J and $250.8 \mathrm{mg} / \mathrm{l}$ lovastatin. Although much further work is required to improve methanol utilization and concomitant production of value-added chemicals, these preliminary studies demonstrate a potential in engineering non-methylotrophic eukaryotic microbes for methanol utilization and scaling their use toward industrial biotechnological processes in the future.

\section{Conclusions}

In the recent years, native methylotrophs such as $M$. extorquens AM1 have gained interest highlighting the important role of methanol as a future alternative carbon feedstock. With understanding of native metabolic pathways and further development of metabolic engineering tools in M. extorquens AM1, it may be feasible to rationally manipulate this microorganism for producing bio-based chemicals and biofuels (Lieven et al., 2018). However, further strain optimization is required to make this system industrially relevant. Alternatively, engineering synthetic methylotrophic bacteria and yeasts provide attractive alternative platforms for methanol conversion. Examples of methanol assimilation by engineered synthetic methylotrophs demonstrate the potential for using traditional industrial microorganism as chassis for production of value-added chemicals from methanol.

\section{Future trends}

Two key roadblocks were identified while attempting to engineer native methylotrophs for methanol conversion. First is the insufficient knowledge on the regulatory mechanisms. For example, engineering even one of the best-studied methylotrophs, M. extorquens, for biotechnological applications requires more knowledge on both the essential genes and their associated regulatory networks. The integrated multiple 'omics' approaches and the genome editing tools that are becoming available for a multitude of organisms, will aid in elucidating the essential sets of proteins-metabolites that control the regulatory networks (Jiang et al., 2013; Diether and Sauer, 2017). Recent discovery of the phosphoribulokinase gene playing an unexpected but essential regulatory role provides one example (Ochsner et al., 2017). The second roadblock is the lack of well-developed genetic tools and genetic elements. Schada von Borzyskowski and collaborators demonstrated that extensive sets of plasmids with different properties are required to allow for 
efficient construction of synthetic operons with different promoter strengths in $M$. extorquens AM1 (Schada von Borzyskowski et al., 2015). Liang and colleagues developed biosensor-assisted transcriptional regulator engineered to control metabolic flux re-distribution between $\mathrm{C} 1$ assimilation and chemicals biosynthesis (Liang et al., 2017a). Future work is necessary to explore regulatory mechanism that may not be directly involved in methanol metabolism, but could potentially improve methanol assimilation. It is also necessary to develop advanced engineering tools, such as promoters and RBS's of different strengths to allow for fine tuning gene expression, as well as the convenient Cre/loxP systems for integrating entire heterologous pathways into the genomes of $M$. extorquens (Liang et al., 2017b) and other potential platforms.

For synthetic methylotrophs, a persisting challenge remains the lack of growth on methanol as the sole carbon and energy source. One limitation is the efficiency of methanol oxidation catalyzed by NADdependent Mdh. So far, the most studied is the Mdh from B. methanolicus, which exhibits low enzyme specificity toward methanol and dependency on an activator protein (Price et al., 2016). Recently, Wu and collaborators characterized a NAD-dependent alcohol dehydrogenase (Mdh2) from Cupriavidus necator that does not require an activator (Wu et al., 2016). Further, they engineered a variant of Mdh2 by using directed molecular evolution that showed a 6-fold increase in $\mathrm{Kcat} / \mathrm{Km}$ for methanol and 10-fold decrease in $\mathrm{Kcat} / \mathrm{Km}$ for 1-butanol than that of Mdh from B. methanolicus. Another limitation is the insufficient flux toward regeneration of Ru5P. Bennett and colleagues demonstrated significant progress towards solving this problem, but further progress is needed (Bennett et al., 2018a). Recently, Cui and colleagues used ARTP mutagenesis to identify a mutant with obvious improvement of methanol tolerance and consumption (Cui et al., 2018). This state-of-the-art technique could also be adapted to evolve current synthetic methylotrophs and screen mutants for increased methanol conversion in the future.

\section{Acknowledgements}

Grant information: This work was supported by the National Natural Science Foundation of China (Grant No. 21776149) and Shandong Provincial Key Research and Development Project, China (2016GSF117026).

\section{References}

Anne, K., Heggeset, T.M.B., Müller, J.E.N., Kupper, C.E., Olha, S., Vorholt, J.A., Ellingsen, T.E., and Trygve, B. (2013). Methylotrophic Bacillus methanolicus encodes two chromosomal and one plasmid Born NAD+dependent methanol dehydrogenase paralogs with

different catalytic and biochemical properties. PLOS ONE 8, e59188.

Anthony, C. (2011). How half a century of research was required to understand bacterial growth on $\mathrm{C} 1$ and $\mathrm{C} 2$ compounds; the story of the serine cycle and the ethylmalonyl-CoA pathway. Sci. Prog. 94, 109-137.

Bennett, R.K., Gonzalez, J.E., Whitaker, W.B., Antoniewicz, M.R., and Papoutsakis, E.T. (2018a). Expression of heterologous non-oxidative pentose phosphate pathway from Bacillus methanolicus and phosphoglucose isomerase deletion improves methanol assimilation and metabolite production by a synthetic Escherichia coli methylotroph. Metab. Eng. 45, 75-85.

Bennett, R.K., Steinberg, L.M., Chen, W., and Papoutsakis, E.T. (2018b). Engineering the bioconversion of methane and methanol to fuels and chemicals in native and synthetic methylotrophs. Curr. Opin. Biotechnol. 50, 81-93.

Bird, C.W., Lynch, J.M., Pirt, F.J., and Reid, W.W. (1971). Steroids and squalene in Methylococcus capsulatus grown on methane. Nature 230, 473-474.

Bringel, F., and Vuilleumier, S. (2017). Metabolic regulation: a master role for ribulose-1,5-bisphosphate in onecarbon assimilation. Curr. Biol. 27, R1127-R1129.

Chistoserdova, L. (2018). Applications of methylotrophs: can single carbon be harnessed for biotechnology? Curr. Opin. Biotechnol. 50, 189-194.

Chistoserdova, L.V., and Lidstrom, M.E. (1994). Genetics of the serine cycle in Methylobacterium extorquens AM1: identification of sgaA and $\mathrm{mtdA}$ and sequences of sgaA, hprA, and mtdA. J. Bacteriol. 176, 1957-1968.

Chistoserdova, L., Gomelsky, L., Vorholt, J.A., Gomelsky, M., Tsygankov, Y.D., and Lidstrom, M.E. (2000). Analysis of two formaldehyde oxidation pathways in Methylobacillus flagellatus KT, a ribulose monophosphate cycle methylotroph. Microbiology 146, 233-238. https://doi.org/10.1099/00221287-146-1-233

Chistoserdova, L., Chen, S.W., Lapidus, A., and Lidstrom, M.E. (2003). Methylotrophy in Methylobacterium extorquens AM1 from a genomic point of view. J. Bacteriol. 185, 2980-2987.

Chistoserdova, L., Kalyuzhnaya, M.G., and Lidstrom, M.E. (2009). The expanding world of methylotrophic metabolism. Annu. Rev.Microbiol.63,477-499.https:// doi.org/10.1146/annurev.micro.091208.073600

Clomburg, J.M., Crumbley, A.M., and Gonzalez, R. (2017). Industrial biomanufacturing: The future of chemical production. Science 355, aag0804.

Cui, J., Good, N.M., Hu, B., Yang, J., Wang, Q. Sadilek, M., and Yang, S. (2016). Metabolomics revealed an association of metabolite changes and defective growth in methylobacterium extorquens AM1 overexpressing ecm during growth on methanol. PLOS ONE 11, e0154043. https://doi.org/10.1371/journal.pone.0154043

Cui, L.Y., Wang, S.S., Guan, C.G., Liang, W.F., Xue, Z.L., Zhang, C., and Xing, X.H. (2018). Breeding of 
methanol-tolerant Methylobacterium extorquens AM1 by atmospheric and room temperature plasma mutagenesis combined with adaptive laboratory evolution. Biotechnol. J. 13, e1700679. https://doi.org/10.1002/ biot.201700679

Dai, Z., Gu, H., Zhang, S., Xin, F., Zhang, W., Dong, W., Ma, J., Jia, H., and Jiang, M. (2017). Metabolic construction strategies for direct methanol utilization in Saccharomyces cerevisiae. Bioresour. Technol. 245, 1407-1412.

Danilov, V.S., and Ismailov, A.D. (1989). Bacterial luciferase as a biosensor of biologically active compounds. Biotechnology 11, 39-78.

de la Torre, A., Metivier, A., Chu, F., Laurens, L.M., Beck, D.A., Pienkos, P.T., Lidstrom, M.E., and Kalyuzhnaya, M.G. (2015). Genome-scale metabolic reconstructions and theoretical investigation of methane conversion in Methylomicrobium buryatense strain 5G(B1). Microb. Cell Fact. 14, 188. https://doi.org/10.1186/s12934015-0377-3

Diether, M., and Sauer, U. (2017). Towards detecting regulatory protein-metabolite interactions. Curr. Opin. Microbiol. 39, 16-23.

Erb, T.J., Berg, I.A., Brecht, V., Müller, M., Fuchs, G., and Alber, B.E. (2007). Synthesis of C5-dicarboxylic acids from $\mathrm{C} 2$-units involving crotonyl-CoA carboxylase/ reductase: the ethylmalonyl-CoA pathway. Proc. Natl. Acad. Sci. U.S.A. 104, 10631-10636.

$\mathrm{Fu}$, Y., Beck, D.A., and Lidstrom, M.E. (2016). Difference in C3-C4 metabolism underlies tradeoff between growth rate and biomass yield in Methylobacterium extorquens AM1. BMC Microbiol. 16, 156. https://doi. org/10.1186/s12866-016-0778-4

Guo, X., and Lidstrom, M.E. (2006). Physiological analysis of Methylobacterium extorquens AM1 grown in continuous and batch cultures. Arch. Microbiol. 186, 139-149. https://doi.org/10.1007/s00203-006-0131-7

Harada, H., Yu, F., Okamoto, S., Kuzuyama, T., Utsumi, R., and Misawa, N. (2009). Efficient synthesis of functional isoprenoids from acetoacetate through metabolic pathway-engineered Escherichia coli. Appl. Microbiol. Biotechnol. 81, 915-925. https://doi.org/10.1007/ s00253-008-1724-7

$\mathrm{Hu}$, B., and Lidstrom, M.E. (2014). Metabolic engineering of Methylobacterium extorquens AM1 for 1-butanol production. Biotechnol. Biofuels 7, 156. https://doi. org/10.1186/s13068-014-0156-0

Hu, B., Yang, Y.M., Beck, D.A., Wang, Q.W., Chen, W.J., Yang, J., Lidstrom, M.E., and Yang, S. (2016). Comprehensive molecular characterization of Methylobacterium extorquens AM1 adapted for 1-butanol tolerance. Biotechnol. Biofuels 9, 84. https://doi.org/10.1186/ s13068-016-0497-y

Jiang, W., Bikard, D., Cox, D., Zhang, F., and Marraffini, L.A. (2013). RNA-guided editing of bacterial genomes using CRISPR-Cas systems. Nat. Biotechnol. 31, 233-239. https://doi.org/10.1038/nbt.2508

Kalyuzhnaya, M.G., Yang, S., Rozova, O.N., Smalley, N.E., Clubb, J., Lamb, A., Gowda, G.A., Raftery, D., Fu, Y., Bringel, F., et al. (2013). Highly efficient methane biocatalysis revealed in a methanotrophic bacterium. Nat. Commun. 4, 2785. https://doi.org/10.1038/ncomms3785

Lee, S.Y., Park, J.H., Jang, S.H., Nielsen, L.K., Kim, J., and
Jung, K.S. (2008). Fermentative butanol production by Clostridia. Biotechnol. Bioeng. 101, 209-228. https:// doi.org/10.1002/bit.22003

Leßmeier, L., Pfeifenschneider, J., Carnicer, M., Heux, S., Portais, J.C., and Wendisch, V.F. (2015). Production of carbon-13-labeled cadaverine by engineered Corynebacterium glutamicum using carbon-13-labeled methanol as co-substrate. Appl. Microbiol. Biotechnol. 99, 10163-10176. https://doi.org/10.1007/s00253015-6906-5

Liang, W.F., Cui, L.Y., Cui, J.Y., Yu, K.W., Yang, S., Wang, T.M., Guan, C.G., Zhang, C., and Xing, X.H. (2017a). Biosensor-assisted transcriptional regulator engineering for Methylobacterium extorquens AM1 to improve mevalonate synthesis by increasing the acetyl-CoA supply. Metab. Eng. 39, 159-168.

Liang, W.F., Sun, M.Y., Cui, L.Y., Zhang, C., and Xing, X.H. (2017b). Cre/loxP-mediated multicopy integration of the mevalonate operon into the genome of Methylobacterium extorquens AM1. Appl. Biochem. Biotechnol. 2, 1-13.

Lidstrom, M.E. (2006). Aerobic Methylotrophic Prokaryotes. In The Prokaryotes, Vol. 2: Ecophysiology and Biochemistry, Dworkin, M., Falkow, S., Rosenberg, E., Schleifer, K.-H., and Stackebrandt, E., eds. (SpringerVerlag, New York, NY), pp. 618-634.

Lieven, C., Herrgård, M.J., and Sonnenschein, N. (2018). Microbial Methylotrophic metabolism: recent metabolic modeling efforts and their applications in industrial biotechnology. Biotechnol.J. 13, e1800011. https://doi. org/10.1002/biot.201800011

Liu, Y., Bai, C., Xu, Q. Yu, J., Zhou, X., Zhang, Y., and Cai, M. (2018a). Improved methanol-derived lovastatin production through enhancement of the biosynthetic pathway and intracellular lovastatin efflux in methylotrophic yeast. Bioresources \& Bioprocessing $5,22$.

Liu, Y., Tu, X., Xu, Q. Bai, C., Kong, C., Liu, Q. Yu, J., Peng, Q. Zhou, X., Zhang, Y., et al. (2018b). Engineered monoculture and co-culture of methylotrophic yeast for de novo production of monacolin $\mathrm{J}$ and lovastatin from methanol. Metab. Eng. 45, 189-199.

Meyer, F., Keller, P., Hartl, J., Gröninger, O.G., Kiefer, P., and Vorholt, J.A. (2018). Methanol-essential growth of Escherichia coli. Nat. Commun. 9, 1508. https://doi. org/10.1038/s41467-018-03937-y

Müller, J.E., Meyer, F., Litsanov, B., Kiefer, P., and Vorholt, J.A. (2015a). Core pathways operating during methylotrophy of Bacillus methanolicus MGA3 and induction of a bacillithiol-dependent detoxification pathway upon formaldehyde stress. Mol. Microbiol. 98, 1089-1100. https://doi.org/10.1111/mmi.13200

Müller, J.E.N., Meyer, F., Litsanov, B., Kiefer, P., Potthoff, E., Heux, S., Quax, W.J., Wendisch, V.F., Brautaset, T., Portais, J.C., et al. (2015b). Engineering Escherichia coli for methanol conversion. Metab. Eng. 28, 190-201.

Nagai, H., Masuda, A., Toya, Y., Matsuda, F., and Shimizu, H. (2018). Metabolic engineering of mevalonate-producing Escherichia coli strains based on thermodynamic analysis. Metab. Eng. 47, 1-9.

Ochsner, A.M., Sonntag, F., Buchhaupt, M., Schrader, J., and Vorholt, J.A. (2015). Methylobacterium extorquens: 
methylotrophy and biotechnological applications. Appl. Microbiol. Biotechnol. 99, 517-534. https://doi. org/10.1007/s00253-014-6240-3

Ochsner, A.M., Christen, M., Hemmerle, L., Peyraud, R., Christen, B., and Vorholt, J.A. (2017). Transposon sequencing uncovers an essential regulatory function of phosphoribulokinase for methylotrophy. Curr. Biol. 27, 2579-2588.e6.

Peyraud, R., Kiefer, P., Christen, P., Massou, S., Portais, J.C., and Vorholt, J.A. (2009). Demonstration of the ethylmalonyl-CoA pathway by using $13 \mathrm{C}$ metabolomics. Proc. Natl. Acad. Sci. U.S.A. 106, 4846-4851. https:// doi.org/10.1073/pnas.0810932106

Peyraud, R., Schneider, K., Kiefer, P., Massou, S., Vorholt, J.A., and Portais, J.C. (2011). Genome-scale reconstruction and system level investigation of the metabolic network of Methylobacterium extorquens AM1. BMC Syst. Biol. 5, 189. https://doi.org/10.1186/17520509-5-189

Price, J.V., Chen, L., Whitaker, W.B., Papoutsakis, E., and Chen, W. (2016). Scaffoldless engineered enzyme assembly for enhanced methanol utilization. Proc. Natl. Acad. Sci. U.S.A. 113, 12691-12696. https://doi. org/10.1073/pnas.1601797113

Rohde, M.T., Tischer, S., Harms, H., and Rohwerder, T. (2017). Production of 2-hydroxyisobutyric acid from methanol by Methylobacterium extorquens AM1 Expressing (R)-3-Hydroxybutyryl Coenzyme A-Isomerizing Enzymes. Appl. Environ. Microbiol. 83, e02622-16.

Rohlhill, J., Sandoval, N.R., and Papoutsakis, E.T. (2017). Sort-seq approach to engineering a formaldehydeinducible promoter for dynamically regulated Escherichia coli growth on methanol. ACS Synth. Biol. 6, 1584-1595. https://doi.org/10.1021/acssynbio.7b00114

Schada von Borzyskowski, L., Remus-Emsermann, M., Weishaupt, R., Vorholt, J.A., and Erb, T.J. (2015). A set of versatile brick vectors and promoters for the assembly, expression, and integration of synthetic operons in Methylobacterium extorquens AM1 and other alphaproteobacteria. ACS Synth. Biol. 4, 430-443. https://doi.org/10.1021/sb500221v

Schada von Borzyskowski, L., Carrillo, M., Leupold, S., Glatter, T., Kiefer, P., Weishaupt, R., Heinemann, M., and Erb, T.J. (2018). An engineered Calvin-Benson-Bassham cycle for carbon dioxide fixation in Methylobacterium extorquens AM1. Metab. Eng. 47, 423-433.

Sirirote, P., Tsuneo, Y., and Shoichi, S. (1988). l-serine production from methanol and glycine with an immobilized methylotroph. J. Ferment. Technol. 66, 291-297.

Sonntag, F., Buchhaupt, M., and Schrader, J. (2014). Thioesterases for ethylmalonyl-CoA pathway derived dicarboxylic acid production in Methylobacterium extorquens AM1. Appl. Microbiol. Biotechnol. 98, 45334544. https://doi.org/10.1007/s00253-013-5456-y

Sonntag, F., Kroner, C., Lubuta, P., Peyraud, R., Horst, A., Buchhaupt, M., and Schrader, J. (2015a). Engineering Methylobacterium extorquens for de novo synthesis of the sesquiterpenoid $\alpha$-humulene from methanol. Metab. Eng. 32, 82-94.

Sonntag, F., Müller, J.E., Kiefer, P., Vorholt, J.A., Schrader,
J., and Buchhaupt, M. (2015b). High-level production of ethylmalonyl-CoA pathway-derived dicarboxylic acids by Methylobacterium extorquens under cobalt-deficient conditions and by polyhydroxybutyrate negative strains. Appl. Microbiol. Biotechnol. 99, 3407-3419. https:// doi.org/10.1007/s00253-015-6418-3

Strom, T., Ferenci, T., and Quayle, J.R. (1974). The carbon assimilation pathways of Methylococcus capsulatus, Pseudomonas methanica and Methylosinus trichosporium (OB3B) during growth on methane. Biochem. J. 144, 465-476.

Whitaker, W.B., Sandoval, N.R., Bennett, R.K., Fast, A.G., and Papoutsakis, E.T. (2015). Synthetic methylotrophy: engineering the production of biofuels and chemicals based on the biology of aerobic methanol utilization. Curr. Opin. Biotechnol. 33, 165-175. https://doi. org/10.1016/j.copbio.2015.01.007

Whitaker, W.B., Jones, J.A., Bennett, R.K., Gonzalez, J.E., Vernacchio, V.R., Collins, S.M., Palmer, M.A., Schmidt, S., Antoniewicz, M.R., Koffas, M.A., et al. (2017). Engineering the biological conversion of methanol to specialty chemicals in Escherichia coli. Metab. Eng. 39, 49-59.

Witthoff, S., Schmitz, K., Niedenführ, S., Nöh, K., Noack, S., Bott, M., and Marienhagen, J. (2015). Metabolic engineering of Corynebacterium glutamicum for methanol metabolism. Appl. Environ. Microbiol. 81, 2215-2225. https://doi.org/10.1128/AEM.03110-14

Woolston, B.M., Roth, T., Kohale, I., Liu, D.R., and Stephanopoulos, G. (2018). Development of a formaldehyde biosensor with application to synthetic methylotrophy. Biotechnol. Bioeng. 115, 206-215. https://doi.org/10.1002/bit.26455

Wu, T.Y., Chen, C.T., Liu, J.T., Bogorad, I.W., Damoiseaux, R., and Liao, J.C. (2016). Characterization and evolution of an activator-independent methanol dehydrogenase from Cupriavidus necator N-1. Appl. Microbiol. Biotechnol. 100, 4969-4983. https://doi.org/10.1007/s00253016-7320-3

Yang, J., Zhang, C.T., Yuan, X.J., Zhang, M., Mo, X.H., Tan, L.L., Zhu, L.P., Chen, W.J., Yao, M.D., Hu, B., Yang, S. (2018). Metabolic engineering of Methylobacterium extorquens AM1 for the production of butadiene precursor. Microb Cell Fact. 17, 194. doi: 10.1186/s12934-018-1042-4.

Yang, Y.M., Chen, W.J., Yang, J., Zhou, Y.M., Hu, B., Zhang, M., Zhu, L.P., Wang, G.Y., and Yang, S. (2017). Production of 3-hydroxypropionic acid in engineered Methylobacterium extorquens AM1 and its reassimilation through a reductive route. Microb. Cell Fact. 16, 179. https://doi.org/10.1186/s12934-017-0798-2

Zhang, L., Xiao, W.H., Wang, Y., Yao, M.D., Jiang, G.Z., Zeng, B.X., Zhang, R.S., and Yuan, Y.J. (2017). Chassis and key enzymes engineering for monoterpenes production. Biotechnol. Adv. 35, 1022-1031.

Zhao, L., Chang, W.C., Xiao, Y., Liu, H.W., and Liu, P. (2013). Methylerythritol phosphate pathway of isoprenoid biosynthesis. Annu. Rev. Biochem. 82, 497-530.https:// doi.org/10.1146/annurev-biochem-052010-100934

Zhu, W.L., Cui, J.Y., Cui, L.Y., Liang, W.F., Yang, S., Zhang, C., and Xing, X.H. (2016). Bioconversion of methanol to value-added mevalonate by engineered Methylobacterium extorquens AM1 containing an optimized mevalonate pathway. Appl. Microbiol. Biotechnol. 100, 2171-2182. https://doi.org/10.1007/s00253-015-7078-z 